\title{
Agglomeration of Ti-SBA-15 with clays for liquid phase olefin epoxidation in a continuous fixed bed reactor.
}

Juan A. Melero, ${ }^{* \dagger}{ }^{\dagger}$ Jose Iglesias, ${ }^{\dagger}$ Javier Sainz-Pardo, ${ }^{\dagger}$ Pilar de Frutos, ${ }^{\dagger}$ Sandra Blázquez ${ }^{\ddagger}$

$\dagger$ Department of Chemical and Environmental Technology, ESCET, Universidad Rey Juan Carlos, C/Tulipán s/n, E-28933 Móstoles, Madrid, Spain

$\ddagger$ Repsol-YPF Research Center, E-28931 Móstoles, Madrid, Spain.

Published on:

Chemical Engineering Journal 139 (2008) 631-641

doi:10.1016/j.cej.2008.01.013

* To whom correspondence should be addressed:

e-mail: juan.melero@urjc.es; tel: +34 91 4887087; fax: +34 914887068 


\begin{abstract}
Titanium-containing SBA-15 material has been agglomerated with bentonite clay to form macroscopic structured catalyst particles with the purpose of being used in continuous epoxidation processes on a fixed bed reactor. The binding conditions, the mass ratio catalyst to binding agent as well as the calcination temperature of the material were studied to improve as much as possible the catalytic behavior and mechanical strength of the titanium-based catalyst. The binded material has been finally tested in the liquid epoxidation of 1-octene with ethyl benzyl hydroperoxide in a continuous up-flow fixed bed reactor. Reaction results reveal a better catalytic performance of this catalyst, both regarding to the conversion of the olefin and efficient use of the oxidant, than conventional commercially available $\mathrm{TiO}_{2}-\mathrm{SiO}_{2}$, specially when pellet surface hydrophobization is performed by silylation treatment.
\end{abstract}

\title{
Keywords
}

Ti-SBA-15; Mesoporous; Epoxidation; Agglomeration; Fixed bed reactor. 


\section{Introduction}

Epoxides are very important in the chemical industry due to their application as intermediates for the synthesis of a large variety of products. For example, propylene oxide is employed as starting material in the synthesis of many commercial products, including adhesives, paints, and cosmetics (1). The major application of propylene oxide is the production of polyether polyols, which are mainly used for the production of polyurethane foams, though other applications include the production of propylene glycol ethers and propylene glycol (2). Current methods for the production of propylene oxide are the chlorohydrin process and the hydroperoxide process (1). The first of them involves a great environmental impact due to the production of chlorinated by-products and calcium chloride wastes which have not commercial value, besides wastewater with high concentration in salts are produced. For many reasons the hydroperoxide process has displaced the chlorhydrin process and now it is the most extended industrial procedure for the production of propylene oxide (1). This process shows low production of waste and good efficiency in the use of the oxidant. However, a great disadvantage is the production of a by-product, ethylbenzyl alcohol or tert-butyl alcohol, depending on which variant of the hydroperoxide process is applied (SMPO / PO-TBA) (1), which has to be commercially valuable to ensure the proficiency of the process. Thus, the use of industrial oxidants like the ethylbenzyl hydroperoxide (EBHP) or tert-butyl hydroperoxide (TBHP) lead to two different process resultant from the valuation of the alcohol by-product. The former leads to ethylbenzyl alcohol which is subsequently transformed in styrene through a dehydration step. The process is known as SMPO because of the name of the two main products - Styrene Monomer Propylene Oxide. On the other hand, the tert-butyl alcohol resultant from TBHP is usually valuated through its conversion into methyl tert-butyl ether (MTBE) which shows commercial interest as fuel additive. In this case the overall process, including the production of propylene oxide, is known as PO-TBA (Propylene Oxide - tert- 
Butyl Alcohol). Nevertheless, SMPO process is more extended than PO-TBA process because of the higher commercial value of styrene compared to tert-butylalcohol (1). Besides the valuation of the alcohol resultant form the conversion of the oxidant, some other difficulties are found for the separation of the residual hydroperoxide from the oxirane. Thus, the hydroperoxides employed in the production of oxiranes have to be highly converted in a very high efficient manner, decreasing in this way the importance of the main disadvantages of the hydroperoxide process. In this sense, the catalyst plays an important role in achieving both objectives. Thus, the development of an efficient catalyst for the production of propylene oxide has been and still it is an important industrial challenge (2). Different kinds of catalysts can be used to drive the oxidation of olefins by alkyl hydroperoxides but most of the industrial processes are based on molybdenum (3),(4) and titanium (5) as catalytic active metal. Moreover, a great part of these epoxidation processes involve the use of molybdenum homogeneous catalysts and therefore the catalyst recovering results difficult as well as the equipment corrosion troubles become frequent. The discoveries of the Shell catalyst (6) and TS-1 zeolitic material (7), as heterogeneous catalysts for olefin epoxidation, partially overcame these troubles and nowadays they are still being used on an industrial scale, although the latter makes use of hydrogen peroxide as oxidant. However, these materials show some inconvenients, such as the high sensitivity of titanium species against water traces in the Shell catalyst or the small pore size of the TS-1 material which hinders the access of bulky substrates to the catalytic sites, reducing the applicability of the material. The oxidation of bulky substrates requires the synthesis of materials with large pores such as mesoporous xerogels (8) or the recently developed mesostructured materials MCM-41 (9) and SBA-15 (10),(11), to be used as catalytic supports (12)-(15). The preparation of Ti-substituted SBA-15 materials has received special attention due to several advantages offered by this material like the open porous structure or the high hydrothermal stability, moreover compared to MCM 
type materials. Incorporation of titanium species into the matrix of SBA-15 type mesostructured materials can be achieved by post-synthesis grafting treatments, involving the reaction between the silica support and a solution containing the titanium starting material (16),(17), and direct synthesis procedures (18). Latter strategy of synthesis usually leads to more stable and dispersed supported metal species than other methods, however its use involves more difficulties mainly caused by the strong acidic conditions for the synthesis of SBA-15 materials. Recently, we have reported that some of the troubles related to the very low $\mathrm{pH}$ value of the synthesis media for the incorporation of the metal species can be partially overcome by a proper selection of the titanium precursor (18). Titanocene dichloride as titanium source and proper synthesis conditions allow to obtain Ti-SBA-15 samples showing a good metal dispersion, free from anatase domains and displaying a high accessibility to the titanium sites (18). These materials have shown a very high catalytic activity in the epoxidation of olefins with alkyl hydroperoxides under batch conditions.

Industrial requirements for catalysts usually involve their adaptability to continuous processes and Ti-SBA-15 materials are usually prepared as fine powders which avoid their use in a continuous flow fixed bed reactor because of the extremely high pressure drop caused by the catalyst. Very few articles deal with the preparation of macroscopically structured particulated catalyst for its use in a continuous process (5),(19),(20). These articles have prompted us to prepare an extruded catalyst based on Ti-SBA-15 which might have interest in a prospective industrial application. Herein, we report our studies on the formulation of an extruded TiSBA-15/bentonite catalyst prepared from the powdered mesostructured material. This material has been fully developed from the early stages to its application in a packed-bed reactor for the liquid-phase epoxidation of 1-octene as model olefin with ethylbenzyl hydroperoxide as oxidant. 


\section{Experimental}

\subsection{Materials and methods}

Titanocene dichloride $\left[\mathrm{Cl}_{2} \mathrm{TiCp}_{2}, 97 \%\right]$ was used as titanium source for the synthesis of titanium functionalized mesostructured materials and purchased from ABCR. Non-ionic surfactant [Pluronic 123, $\mathrm{EO}_{20} \mathrm{PO}_{70} \mathrm{EO}_{20}, \mathrm{M}=5800$ ], tetraethylorthosilicate [TEOS, 98\%], hexamethyldisilazane [HMDS, 99\%] and 1-octene [99\%] were purchased from Aldrich Chemicals. Ethylbenzyl hydroperoxide [EBHP, 50\% wt in ethylbenzene] was kindly provided by Repsol-YPF to be used as oxidant in epoxidation reactions. The oxidant has been employed as received without previous purification. Commercial $\mathrm{TiO}_{2}-\mathrm{SiO}_{2}$ catalyst (Shell type catalyst) was also provided by Repsol-YPF as $2 \mathrm{~mm}$ spherical pellets as catalyst reference (21). This reference material has been widely studied in the epoxidation of different olefins using several oxidation agents, from the simplest hydrogen peroxide to the bulky ethylbenzyl hydroperoxide, showing for all of them good performance in epoxidation reactions and efficient use of the oxidant. Chemical analysis of the material revealed titanium content of $0.96 \%$ in weight basis.

\subsection{Synthesis of powder Ti-SBA-15}

The powder Ti-SBA-15 samples have been prepared accordingly to the method previously described in literature (18). In a typical synthesis, $4 \mathrm{~g}$ of tri-block copolymer were dissolved in $125 \mathrm{~mL}$ of an aqueous solution of $\mathrm{HCl} 0.5 \mathrm{M}$. The resultant mixture was then heated up to $40^{\circ} \mathrm{C}$ before adding the $\mathrm{Cl}_{2} \mathrm{TiCp}_{2}$. The metal species were prehydrolyzed for at least 3 hours, followed by the addition, in a unique step of the silicon precursor (TEOS). The resultant gel was then kept under stirring at $40^{\circ} \mathrm{C}$ for 20 hours and hydrothermally aged at $100^{\circ} \mathrm{C}$ for 24 additional hours under static conditions. The solid was recovered by filtration and air-dried. 
Surfactant was removed by calcination in air at $550^{\circ} \mathrm{C}$ for 5 hours under static conditions. The solid was then recovered as a white fine powder.

\subsection{Macroscopically structured Ti-SBA-15 materials}

Agglomeration of powder Ti-SBA-15 catalysts with clays has been performed using different procedures. All of the methods make use of inorganic agglomerant clay (bentonite or sepiolite) and an organic additive (methyl cellulose, 5\% wt.). Mixing all of the components with ultra pure water leads to a homogeneous mixture which is kneaded to achieve an uniform distribution of the liquid. The processing of the resultant composition varies depending on the agglomeration method:

Method $A$ consists on drying the mixture on air at $110^{\circ} \mathrm{C}$ during 24 hours followed by a crushing step and a final sieving to achieve a material with particle size comprised between 0.7 and $1.0 \mathrm{~mm}$. The resultant particles were then calcined in air under static conditions using a heating ramp of $0.3^{\circ} \mathrm{C} \cdot \mathrm{min}^{-1}$ up to $550^{\circ} \mathrm{C}$ during 2 hours.

Method $B$ comprises an extrusion step of the raw mixture to form rods. The drying step is carried out in a drying chamber equipped with a humidity controller under the following program: First the extruded rods were subjected to $20^{\circ} \mathrm{C}$ and relative humidity of $70 \%$ during 24 hours. Then, the humidity was reduced down to $35 \%$ keeping constant the temperature conditions for 24 hours. Final stage comprises $80^{\circ} \mathrm{C}$ and relative humidity of $10 \%$ during 20 hours. The dried rods were then calcined in air at $550^{\circ} \mathrm{C}$ for 2 hours using a heating ramp of $0.5^{\circ} \mathrm{C} \min ^{-1}$. The final materials were then crushed and sieved to get particles sized between 0.7 and $1.0 \mathrm{~mm}$.

Method $C$ derives from method B. In this procedure, instead of crushing the calcined samples, the catalyst particles are formed before the drying step by cutting the extruded rod in $2 \mathrm{~mm}$ 
length particles. The rest of the stages make use of the same conditions to those described for method B although in this case the crushing step is avoided.

Figure 1 outlines the main steps and final materials obtained through each one of the agglomeration methods.

\subsection{Silylation of agglomerated materials}

The binded Ti-SBA-15/bentonite catalyst was treated with HMDS using a similar procedure to that previously reported in literature (18). In a typical preparation, a suitable amount of freshly agglomerated material was outgassed at $150^{\circ} \mathrm{C}$ during 24 hours under vacuum. The material was then reacted for 24 hours with a dry mixture of toluene/HMDS in a Soxhlet apparatus under $\mathrm{N}_{2}$ atmosphere (mass ratio material/HMDS of 1.0). The treatment was carried out under static conditions in order to avoid particle attrition. The silylated material was then washed several times with toluene a dried under nitrogen atmosphere for $4 \mathrm{~h}$ at $200^{\circ} \mathrm{C}$.

\subsection{Catalyst characterization}

The titanium content of the prepared materials was determined by ICP-atomic emission spectroscopy. In a typical analysis $100 \mathrm{mg}$ were treated with aqueous hydrofluoric acid and diluted to $250 \mathrm{~mL}$ in a calibrated flask. Standard solution of $\mathrm{Ti}\left(1000 \mu \mathrm{g} \cdot \mathrm{L}^{-1}\right)$ was used for the calibration of the apparatus. Solid state ${ }^{29}$ Si MAS-NMR analyses were recorded in a Varian Infinity 400 spectrometer operating at $79.4 \mathrm{MHz}$ under the following conditions: MAS at 6 $\mathrm{kHz} ; \pi / 2$ pulse, $4.5 \mu \mathrm{s}$; repetition delay, $15 \mathrm{~s} ; 3000$ scans. Spectra were referred to tetramethylsilane. The ordering of the mesostructured materials was evaluated by means of XRD analysis performed in a Philips x'pert diffractometer using the $\mathrm{Cu} \mathrm{K} \alpha$ line. The diffraction patterns were collected in the range from $0.6^{\circ}$ to $5.0^{\circ}(2 \theta)$ using a step size of $0.02^{\circ}$ for low angle analysis and in the range from $5.0^{\circ}$ to $50^{\circ}$ with a step size of $0.04^{\circ}$ for high angle 
analysis. $\mathrm{N}_{2}$ adsorption-desorption isotherms were recorded at $77 \mathrm{~K}$ in a Micromeritics Tristar manometric porosimeter. The specific surface areas were calculated using the B.E.T. method whereas the pore size distributions were determined by the application of the B.J.H. method to the adsorption branch of the isotherm using a Harkins-Jura equation for the adsorbed multilayer thickness specially obtained for SBA-15 type materials (22). Thermogravimetric analyses were carried out on a SDT simultaneous 2960 microscale analyzer from TA Instruments. The different contributions to the weight lost have been distinguished through the deconvolution of the DTA signal using Lorentz curves and subsequent integration. Diffuse reflectance ultraviolet spectra (DR-UV-Vis) were collected in a VARIAN CARY-500 spectrophotometer equipped with an integration sphere accessory in the wavelength range from 200 to $600 \mathrm{~nm}$. FTIR analysis were performed using a Mattson infinity series spectrophotometer using the $\mathrm{KBr}$ buffer technique and recording the spectra in the wavenumber range from 4000 to $400 \mathrm{~cm}^{-1}$. XRF analyses were performed on a Philips MagiX spectrometer. The mechanical resistance of agglomerated materials was measured by two different methods, using a standard assay for determining the bulk crushing strength (BCS) (23) and measuring the individual particle resistance. The latter one was carried out as follows: a single particle is subjected to increasing pressure until particle breaks. This procedure is repeated ten times to obtain the mean value.

\subsection{Catalytic epoxidation of 1-octene with ethyl benzyl hydroperoxide}

The catalytic activity of the titanium-functionalized materials has been evaluated in the liquid phase epoxidation of 1-octene with EBHP, using both stirred batch and continuous up-flow fixed bed reactors. The batch catalytic assays were carried out in a two-necked round bottom flask magnetically stirred and immersed in a temperature controlled bath. All reactions were performed at $110^{\circ} \mathrm{C}$ for $2 \mathrm{~h}$. In a typical test both the catalyst and 1-octene (1-octene:catalyst mass ratio of 50.0; mass catalyst of $1.0 \mathrm{~g}$ ) are loaded into the flask and the system is then 
heated up to $110^{\circ} \mathrm{C}$. Once the temperature is reached, the oxidant is added in a unique step by means of a syringe (EBHP: 1-octene molar ratio of 0.12 ) and all the conditions are then kept constant for two hours. The epoxidation reactions performed under continuous flow were carried out in a glass-made fixed bed reactor like that shown in Figure 2. The catalytic fixed bed, $1.2 \mathrm{~cm}$ of inner diameter and $15 \mathrm{~cm}$ length (catalyst loading of $3.0 \mathrm{~g}$ ), is located in a jacketed glassware tube between two beds of spherical glass inert particles. The temperature of the system is controlled $\left(110 / 120^{\circ} \mathrm{C}\right)$ on top of the bed by using an external heating bath recycling a stream of silicone oil through the jacket of the reactor. Typically, the feed solution mixture, containing both 1-octene and oxidant, was loaded into the fixed bed reactor in upflow operation by means of a Gilson 10SC HPLC pump operating at $1.0 \mathrm{~mL} \cdot \mathrm{min}^{-1}$ (calculated spatial time as the weight of catalyst to mass feed flow was of $3.6 \mathrm{~min}$ ). EBHP composition was varied in order to determine the influence of the molar ratio oxidant to substrate in the catalytic behaviour of agglomerated Ti-SBA-15 materials. Under the reaction conditions, epoxide and methyl benzyl alcohol (MBA) as oxidant by-product were the reaction products. In both cases, either using the batch reactor or the fixed bed, the hydroperoxide conversion was calculated by iodometric titration whereas the rest of the reaction products were quantified by gas chromatography analysis.

\section{Results and discussion}

The present contribution is focused on the development of a particulated Ti-SBA-15 catalyst to be used in the liquid phase epoxidation of 1-octene in an up-flow fixed bed reactor. The current research comprises the choice of the binding agent among two clays conventionally employed for the preparation of catalysts, due to their excellent plastic properties: bentonite and sepiolite. Likewise, some parameters such as the calcination temperature, the mass ratio binding agent to powder catalyst and the agglomeration procedure have also been 
investigated. Finally the macroscopically casted catalyst has been tested in the epoxidation of 1-octene in liquid phase in a continuous up-flow fixed bed reactor.

\subsection{Thermal treatment of binding agent}

The choice of the binding agent has been carried out attending to its activity in the decomposition rate of the oxidant after different thermal treatments. Clays are known to show a high concentration of hydroxyl groups responsible for the Brönsted acidity of these materials (24)-(26). These acid centers are the responsible for the non-oxidative consumption of alkyl hydroperoxides (27)-(29). Thus, the selection of the proper binding agent includes also an additional study on the removal of their hydroxyl groups.

Figure 3 shows the TGA analysis carried out for bentonite and sepiolite materials. The detected weight losses have been correlated with different types of hydroxyl groups removed during calcination. Thus, in the case of the bentonite clay(Figure $3 \mathrm{~A}$ ), the mass change detected in the first and second regions, which comprise up to $300^{\circ} \mathrm{C}$, is attributed to the removal of adsorbed water and water molecules located at narrow pores strongly adsorbed onto the surface of the clay. The third region, comprising the range from $300^{\circ} \mathrm{C}$ to $550^{\circ} \mathrm{C}$ includes the removal of the water molecules located between the bentonite plates. Finally, weight loss detected above $550^{\circ} \mathrm{C}$ is assigned to the dehydroxylation of the material by condensation of silanol groups evolving water molecules and leading to a collapse of the clay structure.

On the other hand, sepiolite materials show a different distribution of the weight loss (Figure 3B). TG analysis show up to three different contributions to the total weight loss. The following processes have been assigned to each mass loss (30): The weight change detected up to $110^{\circ} \mathrm{C}$ corresponds to the removal of the moisture adsorbed on the sepiolite surface, the weight loss at $250-275^{\circ} \mathrm{C}$ matches with the water molecules strongly adsorbed filling the 
micropores of the clay structure and finally the rest of the weight loss is attributed to the removal of the solvating water molecules around magnesium ions. Thus, in the temperature range used for this study, no dehydroxilation processes corresponding to the removal of silanol groups has been detected, which is in accordance with previously reported results (30). Higher temperature values were not assayed because of damage of the porous structure of the mesoporous material might be caused in the binded Ti-SBA-15/clay materials (10).

Table 1 shows the weight loss calculated from thermogravimetric analyses carried out on samples of both clays (bentonite and sepiolite), after calcination at different temperatures. For the bentonite material, the contribution of physisorbed water and moisture (regions I and II) to the total weight loss becomes less important insofar the calcination temperature increases. This could be related to the lower affinity of the surface of the clay for water molecules because a decrease on their hydrophylicity. The lower hydrophilic behavior is caused by the lower amount of surface silanol groups being removed by the calcination treatment. Anyway, the contribution to the weight loss never decreases down to zero because there are some water molecules coming from the environment which remain physisorbed onto the surface of these materials. On the other hand, weight loss detected in region III, becomes non significative for samples calcined beyond $550^{\circ} \mathrm{C}$. Unlike water physisorption, this weight loss, as well as that detected for region IV, is not reversible, since the sample does not show any contribution on both regions to the total mass change after rehydration by contact with the atmosphere. Sepiolite has a rather different behavior regarding to the thermogravimetric analysis. Thus, increasing the calcination temperature causes the contribution of the physisorbed water to the total mass loss, especially that located at micropores (region II), became less important. Anyway, the decrease of physisorbed water molecules is not as marked as that detected for bentonite because, for sepiolite materials, the calcination of the different samples is not strong enough to remove the silanol groups and thus, the hydrophilic nature of the clay is not greatly 
modified. On the other hand, rehydration of magnesium ions seems not to be easy once the water molecules have been removed from its coordination sphere, since the contribution of this region (region III) to the total weight loss is not significative after rehydration by contact with atmosphere.

Having in mind, as previously noticed, the quantity of silanol and hydroxyl functionalities located at the surface of the clays is an indirect measurement of their Brönsted acidity, materials calcined at such a high temperature to cause the dehydroxilation of the material by condensation of silanol groups should lead to a lower decomposition extent of alkyl hydroperoxides. In this sense, if the assumed conclusion is right, only the bentonite samples calcined at temperatures above $550^{\circ} \mathrm{C}$, should lead to poor hydroperoxide conversion. Figure 4 shows the results achieved in the EBHP decomposition tests carried out in presence bentonite and sepiolite samples treated at different temperatures. These tests were developed in presence of a $7 \% \mathrm{wt}$. solution of ethylbenzyl hydroperoxide in ethylbenzene for 2 hours at $80^{\circ} \mathrm{C}$.

For both clays, increasing the calcination temperature leads to a gradual decrease of the oxidant consumption, which is in fairly good correlation with the lower quantity of hydroxyl functionalities detected by thermogravimetric and FTIR analyses. In other words, the decrease of the hydroxyl group population onto the surface of the clays causes lower hydroperoxide degradation. Additionally, it is noteworthy for bentonite materials how the hydroperoxide conversion values are rather similar for samples calcined above $550^{\circ} \mathrm{C}$, even for long reaction times. Thus, bentonite samples calcined at $550^{\circ} \mathrm{C}$ and $650^{\circ} \mathrm{C}$ promote EBHP degradation in a similar extention. This is because the silanol groups which are removed at $600^{\circ} \mathrm{C}$ are located in the interlaminar microporous spacing of the bentonite clay, being not accesible by the bulky EBHP and thus they do not catalyze the hydroperoxide decomposition. Thus, although 
samples calcined at $550^{\circ} \mathrm{C}$ and $650^{\circ} \mathrm{C}$ show different content of surface hydroxyl groups, they promote almost the same hydroperoxide consumption.

On the other hand, sepiolite samples cause higher decomposition degrees of the hydroperoxide than those achieved with bentonite materials, whatever the calcination temperature used in the preparation of the samples. Since the elemental analysis by XRF do not provide meaningful differences related to the presence of transition metals able to catalyze the decomposition of hydroperoxides, differences between sepiolite and bentonite in the decomposition of hydroperoxide have been ascribed to the differences in the population of surface hydroxyl groups. Unlike bentonite, sepiolite samples have not been highly dehydroxylated even at such a high temperature as $650^{\circ} \mathrm{C}$. In this sense, all the sepiolite materials tested in this study show some fraction of their original hydroxyl groups content, and thus the clay is still able to catalyze the degradation of EBHP. Therefore, it can be concluded that there is a direct correlation between the decomposition degree of EBHP and the concentration of hydroxyl groups located onto the surface of the clays used in this study. Anyway, increasing the calcination temperature causes a gradual lowering of hydroperoxide conversion, being this effect more accentuated for bentonite materials than for sepiolite samples. Since calcination at $550^{\circ} \mathrm{C}$ is enough to achieve the minimum hydroperoxide conversion with bentonite, this clay, as well as $550^{\circ} \mathrm{C}$ have been selected as binding agent and calcination temperature respectively for the preparation of the agglomerated Ti-SBA-15 materials described in the rest of this research.

\subsection{Composition of the agglomeration raw mixture}

The next variable studied in this research was the proportion of binding agent to catalyst to be employed in the preparation of agglomerated Ti-SBA-15 catalysts. This parameter is essential for the preparation of a catalyst, since it is responsible of two factors: the mechanical strength 
of the final particles and the concentration of catalytic sites in the final material. Thus, a high proportion of binding agent provides high mechanical resistance but the catalytic behaviour of the final material may be greatly reduced because of an excessively low amount of active catalyst. Thus, this study pursues compromising the mass ratio of binding agent to achieve agglomerated materials with high catalytic activity and mechanical resistance. The amount of binding agent has been varied between $10 \%$ and $40 \% \mathrm{wt}$. in the agglomeration raw mixture. The materials were prepared by the agglomeration method A (see experimental section) to achieve a particle size between $0.75 \mathrm{~mm}$ and $1.0 \mathrm{~mm}$.

Figure 5 shows the $\mathrm{N}_{2}$ adsorption isotherms recorded for the different binded Ti-SBA-15 / bentonite catalysts after calcination at $550^{\circ} \mathrm{C}$. The so-prepared materials display type IV isotherms featured with $\mathrm{H} 1$ hysteresis loops, accordingly to the I.U.P.A.C. classification, typical from mesostructured materials with pore sizes larger than $40 \AA$. Increasing the amount of the clay in the agglomeration mixture leads to a clear decrease of the adsorbed nitrogen volume on every partial pressure measurement points. These differences between samples are related to a decreasing in the total pore volume and specific surface area when increasing the amount of bentonite in the final materials (Table 2). The explanation for this behaviour is the simply dilution of the mesostructured material with the bentonite clay, which acts as a dispersant and, since the surface area and pore volume of this mineral is quite low, its contribution to the resultant textural properties is negligible. A clear evidence of this fact is the high coincidence of the relative isotherms of all the materials depicted in Figure 5 B. Regarding to the mesoscopic ordering of the final agglomerated materials, XRD analysis provides diffraction patterns typical from a P6mm structure regardless of the clay content (see supplementary data). Textural properties and XRD patterns indicate that the mesostructure has been well preserved during the agglomeration and thermal treatment of the Ti-SBA15/bentonite bindings. 
Figure 6A depicts the trend of the titanium loading as well as the particle resistance versus the amount of bentonite in the final materials. The use of increasing amounts of binding agent causes a decreasing of the final metal content in the materials because of an expected dilution effect. In contrast, the higher loading of bentonite the more resistant agglomerated particles. In this sense, it seems to be an inflection point of the trend in the particle resistance around a mass composition in bentonite of $25 \%$ - 30\% wt. Higher clay loadings do not provide increasing particle resistance but, on the contrary, causes an unnecessary dilution of the active catalyst reducing in this way the proportion of titanium species. This dilution can exert negative influence on the catalytic behaviour of the agglomerated materials in catalytic epoxidation of olefins. Thus, in order to evaluate properly which is the true influence of the binding between bentonite and Ti-SBA-15 materials, some catalytic assays have been performed using agglomerated materials as catalysts in a stirred batch reactor in presence of EBHP as oxygen source. Figure $6 \mathrm{~B}$ shows the correlation between the bentonite loading and the titanium content with the conversion of the substrate. It is noticeable that all the materials displayed similar values for the efficiency in the use of the oxidant towards the epoxide $(\sim 70$ $75 \%$ ) and moderate oxidant conversion $(60-80 \%)$. These results indicate that the nonoxidative consumption of EBHP has been cut down because of the removal of surface hydroxyl functionalities during post-synthesis calcination. Thus, when increasing the bentonite loading on the final particles of binded Ti-SBA-15/bentonite, the conversion of 1 octene is reduced because of the lower quantity of catalytic sites present in the material. In this sense, it is noteworthy the dependency of the substrate conversion with the titanium loading follows a linear relationship indicating the catalytic activity per titanium site is almost the same for all the materials. This result means that the intrinsic catalytic activity per titanium site of the Ti-SBA-15 materials has not been changed during the agglomeration process and it is independent on the clay content. 
In this context, the optimal amount of bentonite for the preparation of agglomerated Ti-SBA15 materials seems to be ca. $30 \mathrm{wt} \%$. because it leads to an important increasing in the particle resistance whereas the amount of titanium present in the resulting material is not largely reduced. Larger amounts of bentonite do not provide increasing particle mechanical resistance whereas it reduces the activity of the final material because of dilution of the TiSBA-15 catalyst.

\subsection{Enhancement of mechanical resistance and hydrophobicity of catalyst particles.}

The method for producing particulated solids exerts an important influence on the final properties of the casted solid. Thus, several steps are crucial in the final mechanical resistance, for instance the drying method (31). During the drying step, particles trend to shrink and become distorted and cracked reducing the mechanical resistance of the particle. In order to avoid these problems, water has to be slowly removed from extruded particles. Additionally, any procedure involving transmission of mechanical stress to the solid can dramatically reduce the mechanical resistance of the particles because of the creation of micro fissures inside the particles. With the purpose to increase as much as possible the mechanical resistance of the Ti-SBA-15/bentonite materials, different agglomeration methods were used (Figure 1). Thus, the general procedure to obtain particulated Ti-SBA-15/bentonite materials (method A) has been modified through the use of an extrusion step as well as an intermediate controlled drying procedure (method B). Additionally, the extruded rods have been reduced to discrete particulated solids by using a crushing step (method B) or by cutting the moist rods (method C) into particles before drying and calcination step. Table 3 shows the physicochemical properties of the materials obtained with the different agglomeration methods as well as the results achieved in the catalytic epoxidation of 1-octene in presence of EBHP in a stirred batch reactor. The characterization of the materials has been completed by determining the mechanical particle resistance of the prepared samples. The materials 
obtained through the different agglomeration methods do not show significant differences regarding to their textural and structural parameters. In the same sense, all the agglomeration procedures lead to materials showing almost the same catalytic behavior, both regarding to the conversion of the substrate and the efficiency in the use of the hydroperoxide, indicating the modifications introduced on each agglomeration method do not cause differences on the catalytic activity of the final materials. However, differences regarding to the particle resistance are quite noteworthy since controlling the drying step as well as using an extrusion procedure leads particles with increasing mechanical resistance, probably as a consequence of the lower stress caused by the mild conditions used during the controlled drying procedure. The slow removal of water from extruded rods avoids the formation of microscopic cracks in the solid, jeopardizing the particle resistance. Additionally, it is noticeable the significant increasing achieved in the mechanical strength when casting the particles by cutting before drying and calcination (method C) instead of crushing calcined rods (method B). This better behavior is assigned to the lower mechanical stress suffered by particles when applying this method which leads to materials as resistant as the commercial catalysts used as reference material $\left(\mathrm{SiO}_{2} / \mathrm{TiO}_{2}\right)$, as it is inferred from almost the same BCS index achieved for both materials (Table 3).

All the materials display catalytic activity in the epoxidation of 1-octene. The low values achieved for substrate conversion are due to the employed reaction conditions since the substrate is also used as solvent and thus it is present in large excess regarding to the oxidant. For this reason, the conversion of 1-octene has been referred to the maximum stoichiometric value. On the other hand, as negative feature, all the prepared materials, regardless the agglomeration method employed for their preparation, show a rather low efficiency in the use of the oxidant (ca. 70\%). It is well known the removal of surface hydroxyl functionalities reduces hydroperoxide by-reactions like the non-oxidative consumption, improving in this 
way the efficiency in the use of the oxidant (18). For this reason, post-synthesis silylation has been performed on the material prepared through the agglomeration method $\mathrm{C}$, that showing the higher particle resistance, as well as the commercial catalyst based on $\mathrm{SiO}_{2}-\mathrm{TiO}_{2}$. The physico-chemical properties, as well as their behavior in catalytic tests have also been included in Table 3. Although some textural properties, like the surface area and pore volume are partially modified by the silylation process, because of the grafting of trimethyl silyl functionalities onto the inner porosity, the particle resistance is not altered at all, probably as a consequence of the gentle conditions employed for the silylation treatment of agglomerated particles. On the contrary, the efficiency in the use of the oxidant is enhanced when using silylated materials as catalysts. In this case this parameter reaches an acceptable value of ca. $85 \%$ for the silylated Ti-SBA-15 / bentonite material.

\subsection{Liquid phase epoxidation of 1-octene with EBHP in an up-flow fixed bed reactor}

Once the catalyst has been fully developed and characterized, the final agglomerated and silylated materials have been used as catalysts in the catalytic epoxidation of 1-octene with EBHP under liquid phase in a fixed bed reactor. Table 4 summarizes the catalytic results obtained in the epoxidation of 1-octene in a fixed bed reactor under different amounts of the oxidant in the feedstock solution and using the silylated Ti-SBA-15/bentonite material as catalyst. Since the conversion of the oxidant, as well as the efficiency on its use, are critical parameters in the epoxidation of olefins with alkyl hydroperoxides, maximizing the conversion of this reagent in an efficient way can improve the proficiency of the process. In this way, results indicate that increasing the amount of the oxidant in the reaction mixture largely decreases the activity of the catalysts, since the relative conversion achieved for the olefinic substrate decreases from almost $60 \%$ to less than $12 \%$. Nevertheless when considering the real substrate conversion, the reduction of the catalytic activity insofar the oxidant content is increased turns opposite to lead the expected trend. Anyway, when using a 
$12 \%$ mole of oxidant in the feedstock solution, the catalytic activity decreases compared to catalytic assays with a less concentrated oxidant. This fact seems to be related with the observed trend for the efficiency in the use of the oxidant, which is dramatically reduced from $100 \%$ to $60 \%$ when increasing the concentration of EBHP. These results suggest that the secondary reactions involving hydroperoxide, like the non oxidative consumption, are enhanced when increasing the concentration of this reagent in the reactants stream, becoming as important as the epoxidation reaction.

The catalyst developed in this study has been compared with a commercial Shell-type $\mathrm{SiO}_{2-}$ $\mathrm{TiO}_{2}$ catalyst. Results reveal the SBA-based catalyst display a better catalytic behaviour than the commercial one since the olefinic substrate is converted in a higher extension, even containing much lower titanium loading than $\mathrm{TiO}_{2}-\mathrm{SiO}_{2}$ commercial catalyst does. This fact is easily observed when comparing the catalytic activity of both catalysts per gram of catalyst and per gram of titanium (Table 4). The first one indicates a similar catalytic behaviour of both materials per mass of catalysts but differences are clearly evident when comparing the catalytic activity per titanium site. In this way the catalytic activity of the agglomerated TiSBA-15 material is almost twice that calculated for the commercial $\mathrm{TiO}_{2}-\mathrm{SiO}_{2}$ based catalyst. In the same sense, the oxidant is largely converted, almost total conversion in the steady state, when using the commercial catalyst. This behaviour involves a much lower efficiency in the use of the oxidant than that achieved with the mesostructured catalyst. This fact is related to the amount of surface hydroxyl functionalities at the commercial catalyst. A deeper insight to the population of hydroxyl functionalities onto both catalysts, performed by means of solid state MAS-NMR and FTIR analysis (see supplementary data), reveals a much higher amount of these groups located onto the commercial catalysts than inside the pores of the SBA based material. In this way, the non-oxidative consumption of the hydroperoxide is enhanced when using the commercial catalyst. This different behaviour between both materials can be 
explained because of the different composition of both solids. Thus, the commercial catalyst is based on $99 \%$ wt. silica and thus, the amount of hydroxyl functionalities is much higher than that located at the agglomerated material before silylation. Hydrophobization treatment is not so effective in the removal of hydroxyl groups for the commercial catalyst than for the mesostructured material and thus, the behaviour of both treated materials regarding to the efficient use of the hydroperoxide becomes different.

\section{Conclusions}

Mesostructured Ti-SBA-15 material has been agglomerated with bentonite clay to lead macroscopically structured catalyst particles displaying good catalytic activity in the epoxidation of 1-octene with ethylbenzyl hydroperoxide under liquid phase conditions. The calcination temperature of the Ti-SBA-15/bentonite binding as well as the surface hydrophobization of the calcined material by silylation treatment exert a dramatic effect in the improvement of the effective use of the hydroperoxide, minimizing the effect of the non oxidative consumption of the oxidant because of catalytic decomposition. The comparison between this novel structured material with conventional Shell-type $\mathrm{SiO}_{2}-\mathrm{TiO}_{2}$-based catalyst in the epoxidation of 1-octene with ethyl benzyl hydroperoxide in a continuous up-flow fixed bed reactor reveals a much better catalytic behaviour of the mesostructured material, not only because of driving higher conversions of the olefin but also because of a more efficient use of the oxidant. This new extruded Ti-SBA-15-based material might have true industrial potential as alternative to the conventional homogeneous catalysts used for the liquid phase epoxidation of olefins under continuous flow conditions. 


\section{References}

(1) T.A. Nijhuis, M. Makkee, J.A. Moulijn, B.M. Weckhuysen, The production of propene oxide: catalytic processes and recent developments. Ind. Eng. Chem. Res. 45 (2006) 34473459.

(2) E. Sacaliuc, A.M. Beale, B.M. Weckhuysen, T.A. Nijhuis, Propene epoxidation over Au/Ti-SBA-15 catalysts. J. Catal. 248 (2007) 235-248.

(3) R.M. Calvente, J.M. Campos-Martín, J.L.G. Fierro, Effective homogeneous molybdenum catalyst for linear terminal alkenes epoxidation with organic hydroperoxide. Catal. Commun. 3 (2002) 247-251.

(4) S.V. Kotov, E. Balbolov, Comparative evaluation of the activity of some homogeneous and polymeric catalysts for the epoxidation of alkenes by organic hydroperoxides. J. Mol. Catal. A General 176 (2001) 41-48.

(5) J.K.F. Buijink, J.J.M. van Vlaandern, M. Crocker, F.G.M. Niele, Propylene epoxidation over titanium-on-silica catalysts. The heart of the SM-PO process. Catal. Today 93-95 (2004) 199-204.

(6) F. Wattimena, H.P. Wulff, Shell Oil, A process for epoxidizing olefins with organic hydroperoxides. Br. Pat. 1.249.079, 1971.

(7) M. Taramasso, G. Perego, B. Notari, Snamprogetti S.P.A., Preparation of porous crystalline synthetic material comprised of silicon and titanium oxides. US Pat. 4.410.501, 1982.

(8) C.J. Brinker, G.W. Scherer, Sol-gel science: the physics and chemistry of sol-gel processing, Academic Press, San Diego, 1990. 
(9) C.T. Kresge, M.E: Leonowicz, W.J. Roth, J.C. Vartuli, J.S. Beck, Ordered mesoporous molecular sieves synthezised by a liquid crystal template mechanism. Nature 359 (1992) 710712.

(10) D. Zhao, J. Feng, Q. Huo, N. Melosh, G.H. Fredrickson, B.F. Chmelka, G.D. Stucky, Triblock copolymer syntheses of mesoporous silica with periodic 50 to 300 angstrom pores. Science 279 (1998) 548-552.

(11) D. Zhao, Q. Huo, J. Feng, B.F. Chmelka, G.D. Stucky, Non-ionic triblock and star diblock copolymer and oligomeric surfactant syntheses of highly ordered, hydrothermally stable, mesoporous silica structures. J. Am. Chem. Soc. 120 (1998) 6024-6036.

(12) A. Corma, M.T. Navarro, J. Pérez-Pariente, Synthesis of an ultralarge pore titanium silicate isomorphous to MCM-41 and its application as a catalyst for selective oxidation of hydrocarbons. J. Chem. Soc., Chem. Commun. (1994) 147.

(13) M. Widdenmyer, S. Grasser, K. Köhler, R. Anwander, TiOx overlayers on MCM-48 silica by consecutive grafting. Microporous Mesoporous Mater. 44-45 (2001) 327-336.

(14) Z. Luan, L. Kevan, Characterization of titanium-containing mesoporous silica molecular sieve SBA-15 and generation of paramagnetic hole and electron centers. Microporous Mesoporous Mater. 44-45 (2001) 337-344.

(15) B.L. Newalkar, J. Orlanrewaju, S. Komarneni, Direct Synthesis of titanium-substituted mesoporous SBA-15 molecular sieve under microwave-hydrotermal conditions. Chem. Mater. 13 (2001) 552-557.

(16) T. Maschmeyer, F. Rey, G. Sankar, J.M. Thomas, Heterogeneous catalysts obtained by grafting metallocene complexes onto mesoporous silica. Nature 378 (1995) 159-162. 
(17) T. Tatsumi, K.A. Koyano, N. Igarashi, Remarkable activity enhancement by trimethylsilylation in oxidation of alkenes and alkanes with $\mathrm{H}_{2} \mathrm{O}_{2}$ catalyzed by titaniumcontaining mesoporous molecular sieves. Chem. Commun. (1998) 325.

(18) J.A. Melero, J. Arsuaga, P. de Frutos, J. Iglesias, J. Sainz, S. Blazquez, Direct synthesis of titanium-substituted mesoestructured materials using non-ionic surfactants and titanocene dichloride. Microporous Mesoporous Mater. 86 (2005) 364-373.

(19) G. Chandrasekar, M. Hartmann, M. Palanichamy, V. Murugesan, Extrusion of AlSBA15 molecular sieves: An industrial point of view. Catat. Commun. 8 (2007) 457-461.

(20) F. Martínez, J.A. Melero, J.A. Botas, M.I. Pariente, R. Molina, Treatment of phenolic effluents by catalytic wet hydrogen peroxide oxidation over $\mathrm{Fe}_{2} \mathrm{O}_{3} / \mathrm{SBA}-15$ extruded catalysts in a fixed bed reactor. Ind. Eng. Chem. Res. 46 (2007) 4396-4405.

(21) P. de Frutos, J.M. Campos, Repsol Química S.A. US Patent 6.160.138, 2000.

(22) R. van Grieken, G. Calleja, G.D. Stucky, J.A. Melero, R.A. García, J. Iglesias, Langmuir 19 (2003) 3966-3973.

(23) ASTM D7084-04. Standard test method for determination of bulk crush strength of catalysts and catalyst carriers.

(24) S. Akyüz, T. Akyüz, A.E. Yakar, FT-IR spectroscopic investigation of adsorption of 3aminopyridine on sepiolite and montmorillonite from Anatolia. J. Mol. Struct. 565-566 (2001) 487-491.

(25) M. Akçay, FT-IR spectroscopic investigation of the adsorption pyridine on the raw sepiolite and Fe-pillared sepiolite from Anatolia. J. Mol. Struct. 694 (2004) 21-26. 
(26) J. Billingham, C. Breen, J. Yarwood, In situ determination of Brönsted/Lewis acidity on cation-exchanged clay mineral surfaces by ATR-IR. Clay Minerals 31 (1996) 513-522.

(27) J. Xu, B.L. Mojet, J.G. van Ommen, L. Lefferts, Effects of Brönsted acidity in the mechanism of selective oxidation of propane to acetone on $\mathrm{CaY}$ zeolite at room temperature. J. Catal. 232 (2005) 511-423.

(28) R. Selvin, G.R. Rajarajeswari, L.S. Roselin, V. Sadasivam, B. Sivasankar, K. Rengaraj, Catalytic decomposition of cumene hydroperoxide into phenol and acetone. Appl. Catal. A. 219 (2001) 125-129.

(29) K.-T. Li, P.-H. Lin, S.-W. Lin, Preparation of $\mathrm{Ti} / \mathrm{SiO}_{2}$ catalysts by chemical vapour deposition method for olefin epoxidation with cumene hydroperoxide. Appl. Catal. A. 301 (2006) 59-65.

(30) S. Balci, Thermal decomposition of sepiolite and variations in pore structure with and without acid pre-treatment. J. Chem. Tech. Biotechnol. 66 (1996) 72-78.

(31) L.Y. Lee, S.P. Perera, B.D. Crittenden, S.T. Kolaczkowski, Manufacture and characterisation of silicalite monoliths, Adsorpt. Sci. Technol. 18 (2000) 147-170. 
Table 1. Weight loss contributions in TG analysis performed on bentonite and sepiolite clays calcined at different temperatures. Values in wt $\%$.

\begin{tabular}{|c|c|c|c|c|c|c|c|c|}
\hline \multicolumn{5}{|c|}{ Bentonite } & \multicolumn{4}{|c|}{ Sepiolite } \\
\hline $\mathrm{T}\left({ }^{\circ} \mathrm{C}\right)$ & Reg I & Reg II & Reg III & Reg IV & $\mathrm{T}\left({ }^{\circ} \mathrm{C}\right)$ & Reg I & Reg II & Reg III \\
\hline r.t. & 1.51 & 1.53 & 2.13 & 1.67 & r.t. & 1.43 & 3.69 & 3.07 \\
\hline 300 & 0.69 & 1.40 & 2.07 & 1.69 & 300 & 1.11 & 2.53 & 2.82 \\
\hline 400 & 0.60 & 1.17 & 2.08 & 1.69 & 400 & 0.99 & 1.20 & 2.32 \\
\hline 550 & 0.40 & 1.07 & 0.10 & 1.53 & 550 & 1.02 & 1.93 & 0.64 \\
\hline 650 & 0.30 & 1.14 & 0.19 & 0.48 & 650 & 1.09 & 1.51 & 0.06 \\
\hline
\end{tabular}


Table 2. Physicochemical properties of Ti-SBA-15 / bentonite materials

\begin{tabular}{ccccc}
\hline Sample & $\begin{array}{c}\text { Bentonite } \\
(\mathbf{\%})\end{array}$ & $\begin{array}{c}\mathbf{S}_{\text {B.E.T. }}{ }^{\mathbf{b}} \\
\left(\mathbf{m}^{2} \cdot \mathbf{g}^{-1}\right)\end{array}$ & $\begin{array}{c}\mathbf{V p}^{\mathbf{c}} \\
\left(\mathbf{c m}^{\mathbf{3}} \cdot \mathbf{g}^{-1}\right)\end{array}$ & $\begin{array}{c}\mathbf{D p}^{\mathbf{d}} \\
(\AA)\end{array}$ \\
\hline Ti-SBA-15 & 0 & 809 & 0.95 & 92 \\
S-1 & 10 & 620 & 0.81 & 85 \\
S-2 & 20 & 546 & 0.72 & 85 \\
S-3 & 30 & 447 & 0.64 & 88 \\
S-4 & 40 & 403 & 0.59 & 90 \\
\hline
\end{tabular}

${ }^{a}$ Bentonite composition in weight; ${ }^{\mathrm{b}}$ Specific surface area calculated by the B.E.T. method; ${ }^{c}$ Total pore volume measured by $\mathrm{N}_{2}$ adsorption at $\mathrm{P} / \mathrm{P}_{0}=0.985 ;{ }^{\mathrm{d}}$ Pore size calculated by the B.J.H. method using the K.J.S. correction 
Table 3. Physicochemical properties and catalytic activity of materials agglomerated by different methods

\begin{tabular}{|c|c|c|c|c|c|c|c|c|c|c|}
\hline \multicolumn{2}{|c|}{ Synthesis treatments } & \multicolumn{5}{|c|}{ Physico-chemical properties } & \multicolumn{4}{|c|}{ Catalytic activity $^{\text {a }}$} \\
\hline $\begin{array}{l}\text { Agglomeration } \\
\text { method }\end{array}$ & $\begin{array}{c}\text { Post- } \\
\text { synthesis } \\
\text { treatment }\end{array}$ & $\begin{array}{l}\mathbf{T i}^{\mathbf{b}} \\
(\%)\end{array}$ & $\begin{array}{l}\text { S }_{\text {B.E.T. }}{ }^{c} \\
\left(\mathrm{~m}^{2} \cdot \mathrm{g}^{-1}\right)\end{array}$ & $\underset{\left(\mathrm{cm}^{3} \cdot \mathrm{g}^{-1}\right)}{d}$ & $\begin{array}{l}\mathrm{Dp}^{\mathrm{e}} \\
(\AA \AA \AA)\end{array}$ & $\begin{array}{c}\mathrm{Rp}^{\mathrm{f}} \\
(\mathrm{MPa})\end{array}$ & $\begin{array}{c}X_{\text {OCT }}^{g} \\
(\%)\end{array}$ & $\begin{array}{c}X_{\text {oct }}^{\text {h }} \\
(\%)\end{array}$ & $\begin{array}{c}\mathrm{X}_{\text {EBHP }}{ }^{\mathrm{i}} \\
(\%)\end{array}$ & $\begin{array}{c}\eta_{\text {EBHP }}{ }^{j} \\
(\%)\end{array}$ \\
\hline Method A & --- & 0.57 & 407 & 0.64 & 88 & 2.52 & 3.45 & 63.9 & 91.1 & 70.1 \\
\hline Method B & --- & 0.52 & 407 & 0.62 & 87 & 3.13 & 3.52 & 65.1 & 90.4 & 72.0 \\
\hline Method C & --- & 0.59 & 403 & 0.62 & 86 & $5.94(0.74)$ & 3.42 & 63.3 & 91.5 & 69.2 \\
\hline $\begin{array}{l}\text { Commercial } \\
\mathrm{TiO}_{2}-\mathrm{SiO}_{2}\end{array}$ & --- & 0.96 & 334 & 0.87 & 160 & $5.90(0.72)$ & 3.44 & 63.7 & 85.6 & 74.4 \\
\hline Method C & Silylation & 0.53 & 365 & 0.55 & 80 & 5.94 & 3.24 & 60.0 & 70.4 & 85.2 \\
\hline $\begin{array}{c}\text { Commercial } \\
\mathrm{TiO}_{2}-\mathrm{SiO}_{2}\end{array}$ & Silylation & 0.86 & 294 & 0.85 & 154 & 5.90 & 3.41 & 63.1 & 78.7 & 80.2 \\
\hline
\end{tabular}

${ }^{\text {a }}$ Reaction runs carried out in a batch reactor at $110^{\circ} \mathrm{C}$ for 4 hours. Mass of catalyst $1.0 \mathrm{~g}$. EBHP to 1-octene molar ratio of 0.12. ${ }^{\mathrm{b}}$ Mass titanium loading in final materials. ${ }^{\mathrm{c}}$ Surface area calculated by the method B.E.T. ${ }^{\mathrm{d}}$ Total pore volume measured at $\mathrm{p} / \mathrm{p}_{0}=0.985$. ${ }^{\mathrm{e}}$ Pore size calculated by the BJH method. ${ }^{\mathrm{f}}$ Individual mechanical particle resistance, BCS in brackets. ${ }^{\mathrm{g}}$ Conversion of 1-octene; ${ }^{\mathrm{h}}$ Relative conversion of 1-octene referred to the maximum stoichiometric value. ${ }^{\mathrm{i}}$ Absolute conversion of ethyl benzyl hydroperoxide. ${ }^{\mathrm{j}}$ Efficiency in the use of the oxidant. 
Table 4. Steady state results for the epoxidation of 1-octene in a fixed bed reactor under continuous flow.

\begin{tabular}{|c|c|c|c|c|c|c|c|c|}
\hline Run & Catalyst & $\begin{array}{c}\text { EBHP }^{\mathrm{a}} \\
(\%)\end{array}$ & $\mathbf{X}_{\text {OCT }}^{b}$ & $\begin{array}{c}X_{\text {OCT }} \\
(\%)\end{array}$ & $\mathbf{X}_{\text {EBHP }}{ }^{d}$ & $\eta_{\text {EBHP }}^{\mathrm{e}}$ & $\underset{\left(\mathrm{mmol}^{-} \mathrm{g}_{\mathrm{cat}}^{-1} \cdot \mathbf{h}^{-1}\right)}{\mathrm{f}^{\mathrm{f}}}$ & $\underset{\left(\mathrm{mmol}^{\circ} \mathrm{g}_{\mathrm{Ti}}{ }^{-1} \cdot \mathbf{h}^{-1}\right)}{\mathrm{A}_{\mathrm{T}}^{\mathrm{g}}}$ \\
\hline 1 & \multirow{4}{*}{$\begin{array}{c}\text { Ti-SBA-15 / Bentonite } \\
\text { Silylated }\end{array}$} & 1.5 & 1.5 & 56.5 & 57.8 & 97.8 & 1.02 & 192 \\
\hline 2 & & 3.0 & 3.0 & 54.3 & 62.0 & 87.6 & 1.97 & 372 \\
\hline 3 & & 6.0 & 3.2 & 28.6 & 33.0 & 86.8 & 2.08 & 392 \\
\hline 4 & & 12.0 & 2.5 & 11.7 & 19.0 & 61.5 & 1.69 & 319 \\
\hline 5 & $\begin{array}{c}\text { Commercial } \mathrm{TiO}_{2}-\mathrm{SiO}_{2} \\
\text { silylated }\end{array}$ & 3.0 & 2.5 & 47.3 & 96.4 & 49.1 & 1.71 & 199 \\
\hline
\end{tabular}

${ }^{a}$ Amount of ethyl benzyl hydroperoxide in the feed stream in weight basis; ${ }^{b}$ Conversion of 1-octene; ${ }^{c}$ Relative conversion of 1-octene referred to the maximum stoichiometric value; ${ }^{d}$ Absolute conversion of ethyl benzyl hydroperoxide; ${ }^{e}$ Efficiency in the use of the oxidant; ${ }^{f}$ Catalytic activity in mmol of epoxide per gram of catalyst per hour of reaction; ${ }^{g}$ Catalytic activity in mmol of epoxide per gram of titanium per hour of reaction. 


\section{Figure Captions}

Figure 1. Agglomeration methods for the preparation of macroscopically structured TiSBA-15 materials

Figure 2. Scheme of the up-flow fixed bed reactor for the epoxidation of 1-octene in liquid phase under continuous flow.

Figure 3. Thermogravimetric analysis and weight losses assignation for A) bentonite and B) sepiolite clays.

Figure 4. EBHP decomposition over bentonite and sepiolite clays after different thermal treatments.

Figure 5. Nitrogen adsorption-desorption isotherms recorded at $77 \mathrm{~K}$ for agglomerated TiSBA-15 materials with different loadings of bentonite. A) Recorded scale and B) Normalized scale.

Figure 6. Correlation between the bentonite loading and A) particle mechanical resistance and titanium loading and B) catalytic activity in the liquid phase epoxidation of 1-octene for different clay loadings. (1) conversion of 1-octene referred to the maximum stoichiometric value. 


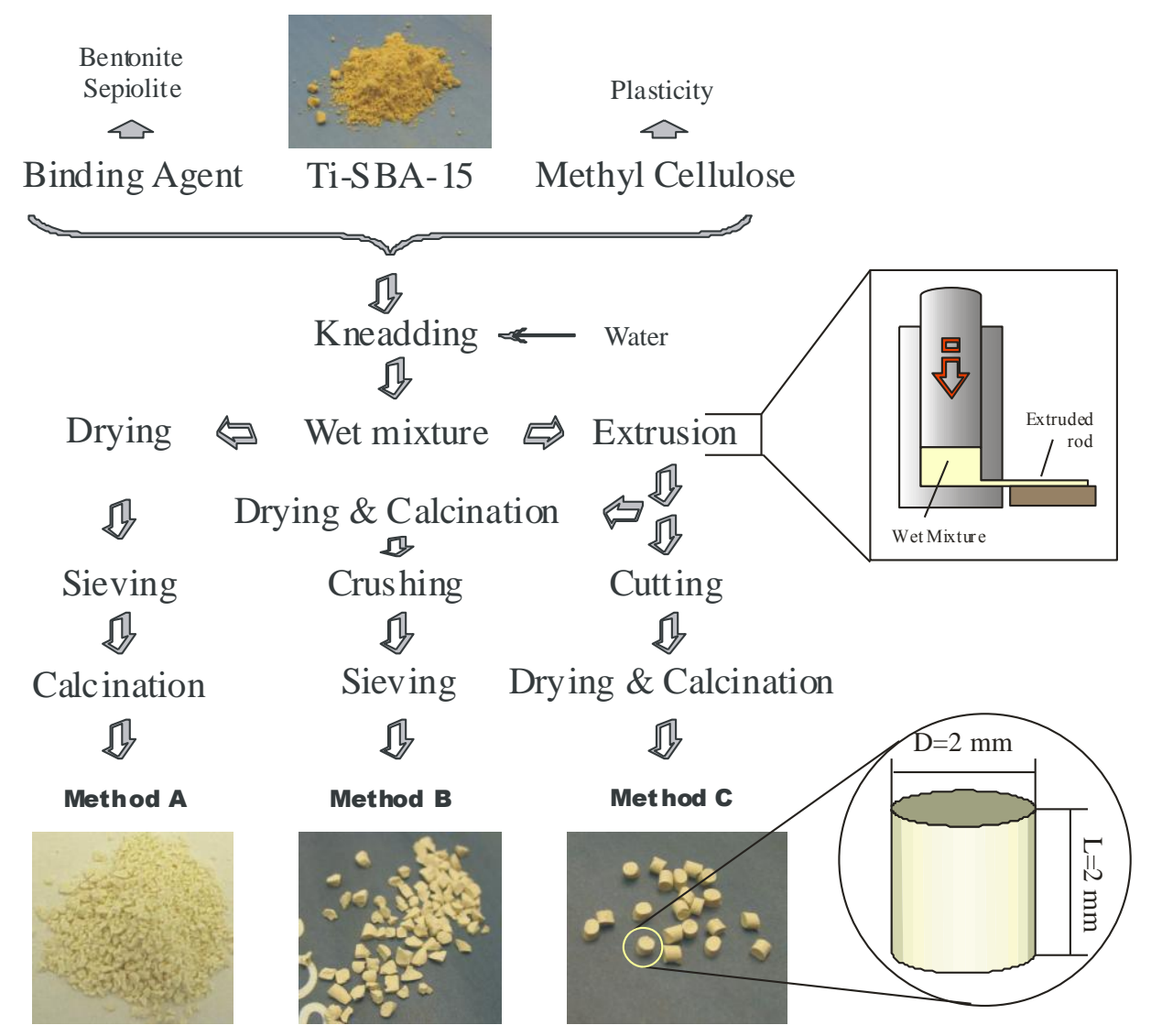

Figure 1

J.A. Melero et al. 
FIXED BED REACTOR FOR LIQUID PHASE OLEFIN EPOXIDATION
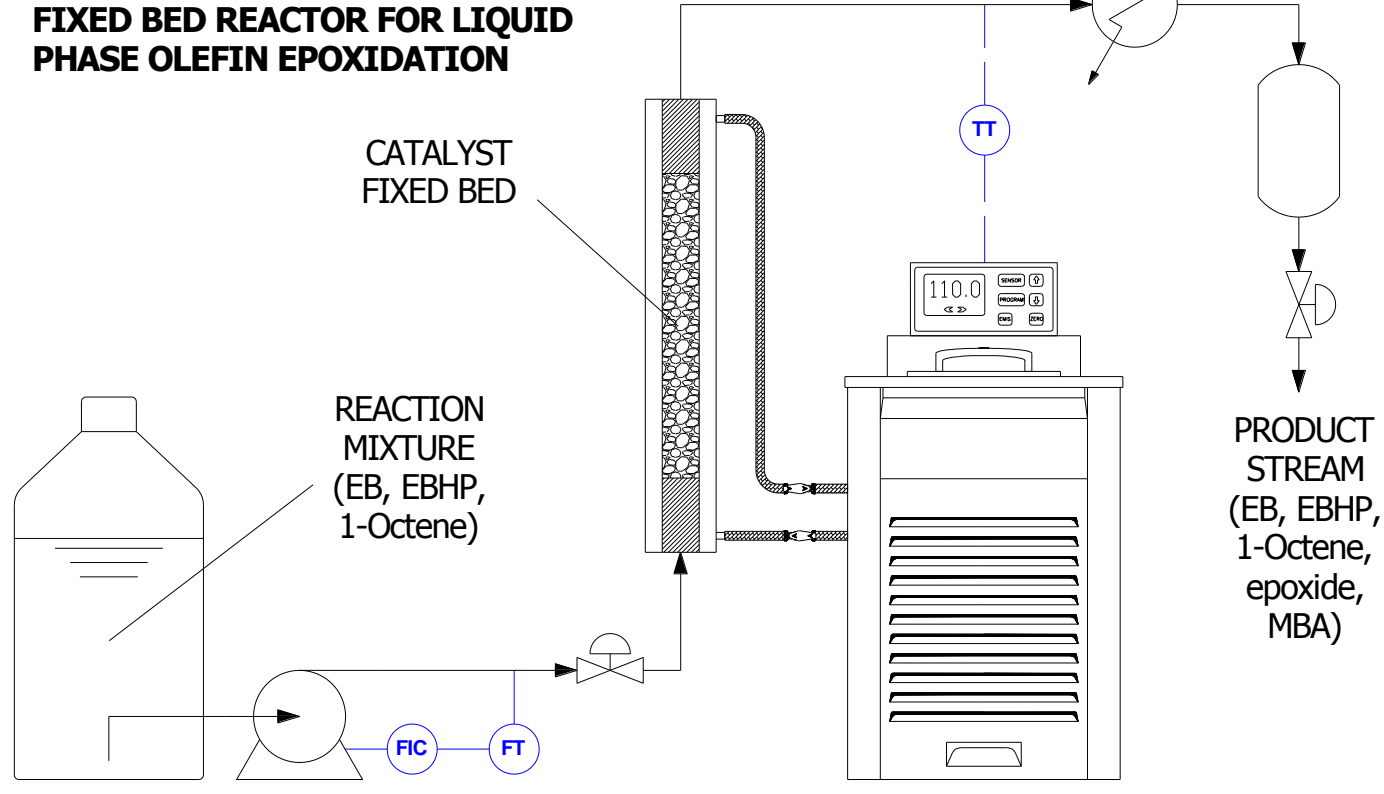

Figure 2

J.A. Melero et al. 

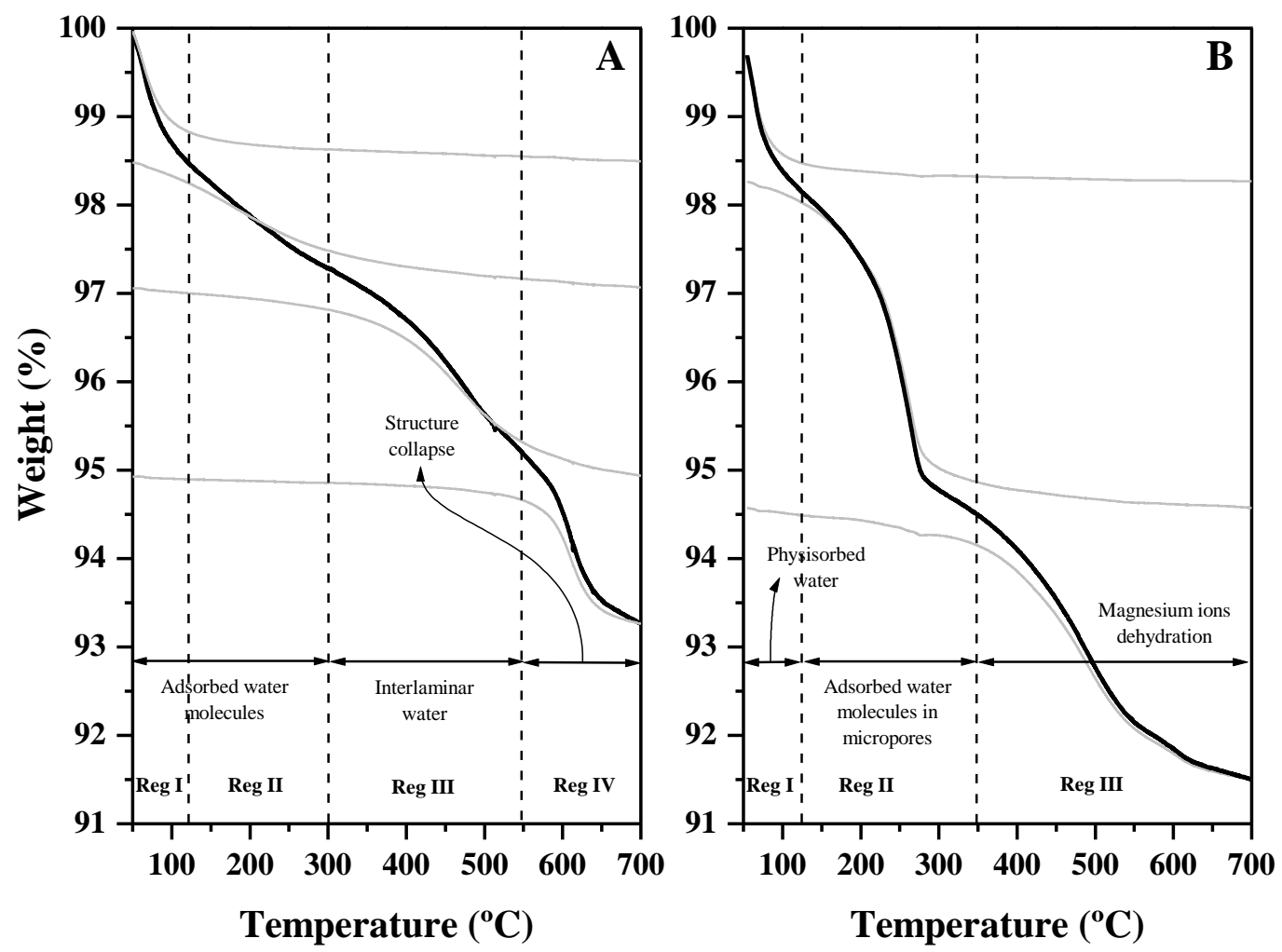

Figure 3

J.A. Melero et al. 


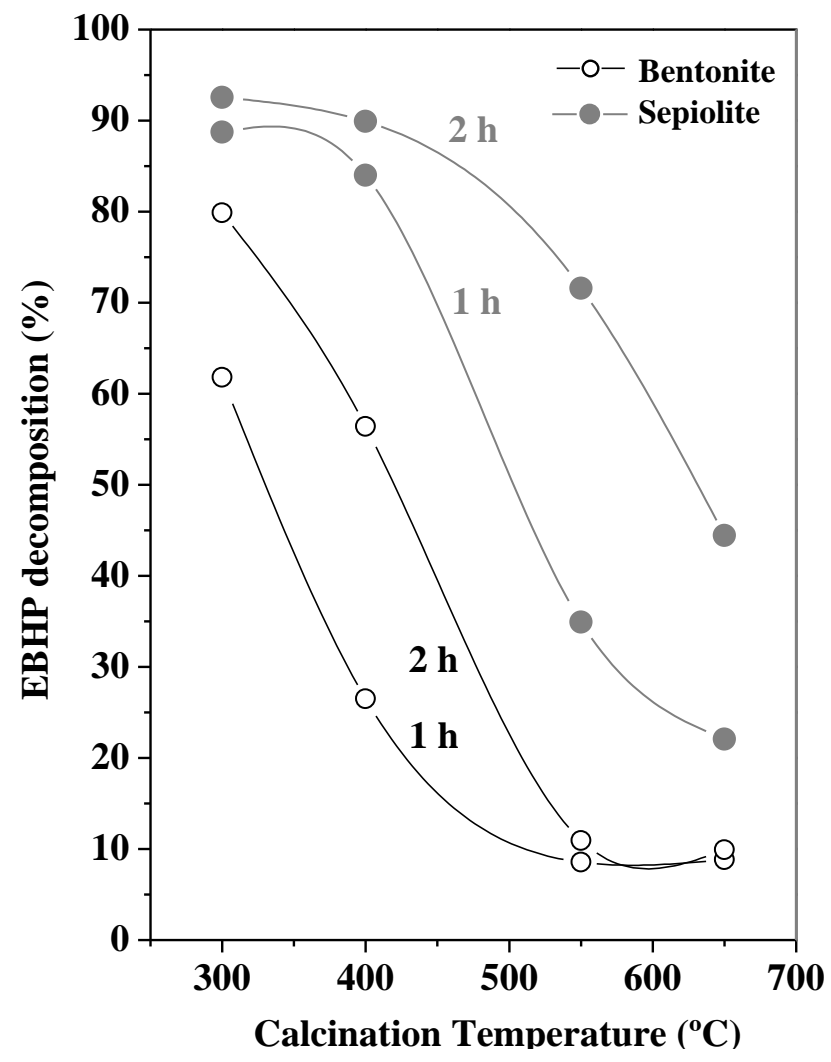

Figure 4

J.A. Melero et al. 

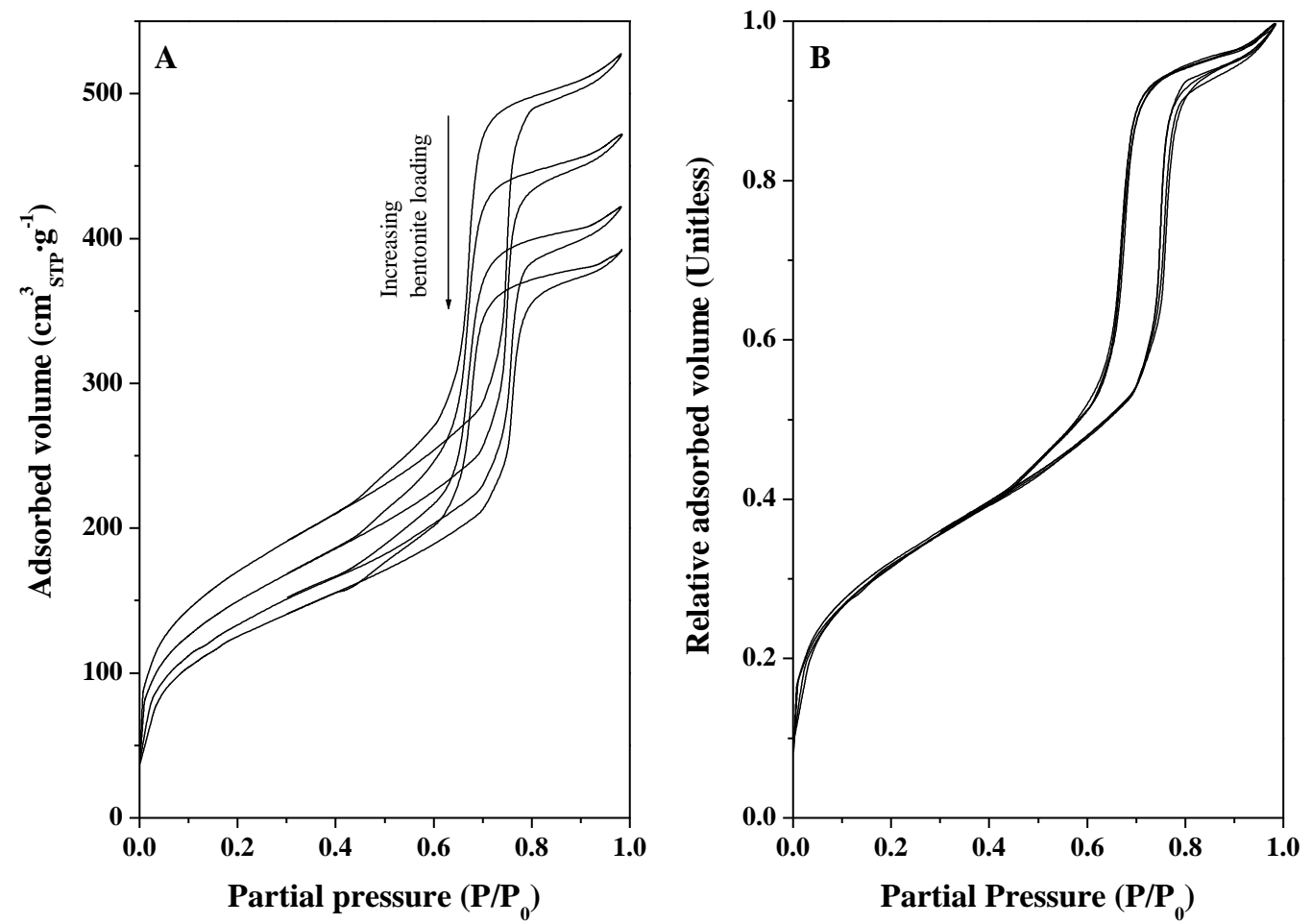

Figure 5

J.A. Melero et al. 

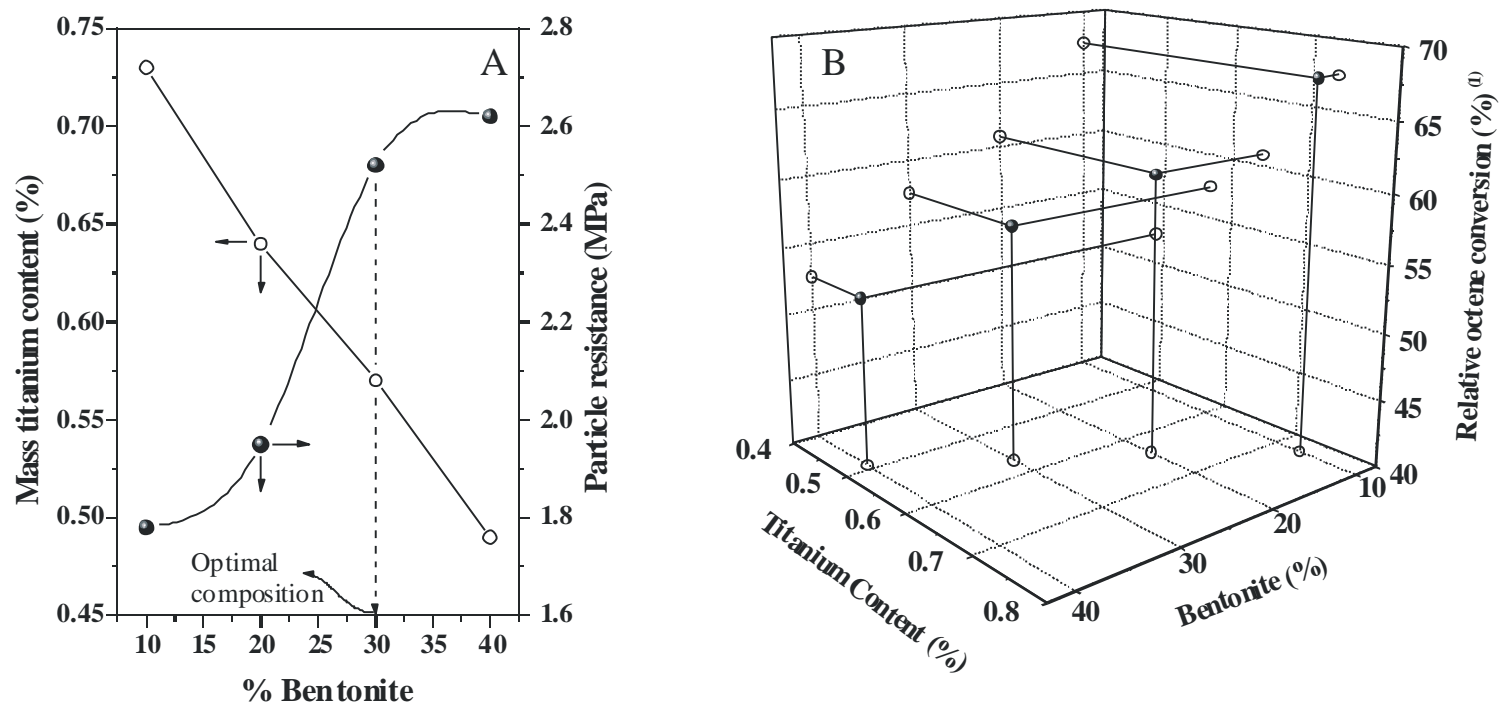

Figure 6

J.A. Melero et al. 